\title{
La sacralisation du pouvoir chez les Minyanka du
} Mali

Making Power Sacred among the Minyanka (Mali)

Danielle Jonckers

\section{OpenEdition}

1 Journals

Édition électronique

URL : http://journals.openedition.org/span/919

DOI : 10.4000/span.919

ISSN : 2268-1558

Éditeur

École pratique des hautes études. Sciences humaines

\section{Édition imprimée}

Date de publication : 1 décembre 1990

Pagination : 145-167

ISSN : 0294-7080

\section{Référence électronique}

Danielle Jonckers, "La sacralisation du pouvoir chez les Minyanka du Mali », Systèmes de pensée en Afrique noire [En ligne], 10 | 1990, mis en ligne le 01 juillet 2013, consulté le 01 mai 2019. URL : http:// journals.openedition.org/span/919; DOI : 10.4000/span.919 


\title{
LA SACRALISATION DU POUVOIR CHEZ LES MINYANKA DU MALI
}

\author{
par
}

\section{Danielle Jonckers}

Religion et politique sont intimement liées chez les Minyanka du Mali. Cette société sans Etat où les confréries initiatiques sont le creuset de la vie politique présente des formes plus ou moins marquées de sacralisation du pouvoir.

Le chef de terre (ninge $f\left(l_{\vartheta}\right.$ ), aîné de la famille du premier occupant présumé' du village, préside à la vie rituelle des communautés non islamisées. Il était autrefois également chef de village (kulo folq). Cette charge est aujourd'hui généralement dévolue à un représentant du gouvernement malien dont l'autorité laïque se distingue de celle des chefs traditionnels, d'essence religieuse.

On attribue au chef de terre un pouvoir sur la nature. Sa vie est entourée d'interdits et l'état de sa santé est censé avoir des répercussions sur la prospérité du village. Cette sacralité est nettement accentuée dans le nord du pays où les rites qui marquent l'avènement à la chefferie de l'aîné des familles fondatrices de certains

\footnotetext{
- Traditionnellement, les conquérants respectaient les prérogatives religieuses des chefs de terre corme on le note dans les sources anciennes (Tarikh el Fattach, 16e-17e siècle, édit. 1913, et Monteil, 1929). Néanmoins il n'est pas rare que les descendants de ces chefs politiques usur-pateurs se présentent aujourd'hui come "maîtres du sol", tirant parti de l'argument d'autochtonie pour perpétuer leur autorité dans de nouvelles conditions historiques ainsi que le soulignent J. Bazin (1988) et J.L. Anselle (1990) dans leurs recherches sur la région de Ségou.
}

Systèmes de pensée en Afrique noire, 10, 1990 


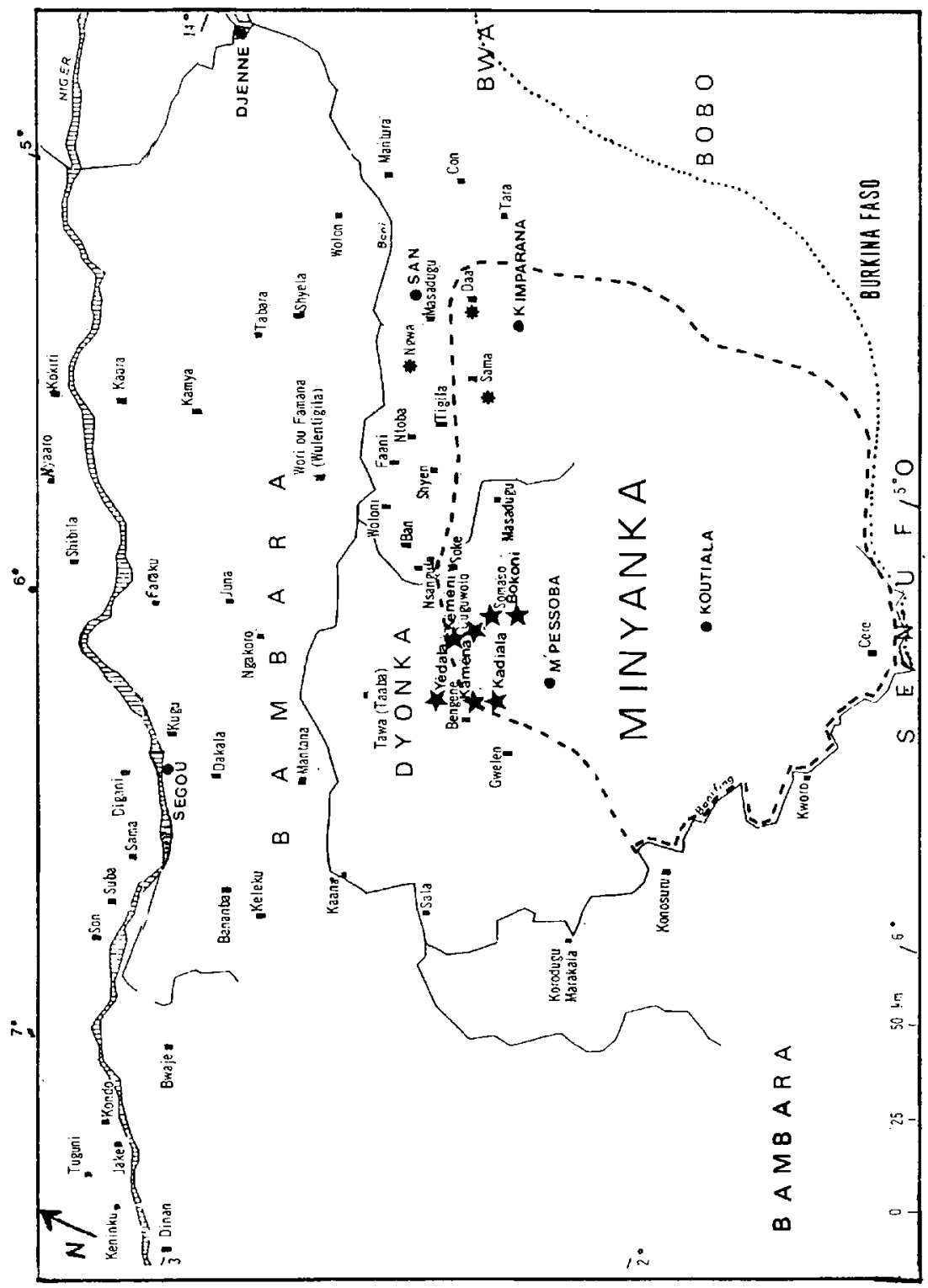

Carte des "villages royaux" de la région de Segou d'après J. Bazin (1988: 385) et des villages minyanka reconnaissant un chef sacré.

- Villages royaux mentionnés par J. Bazin

* Villages de chefs sacrés appelés Tangbula

* Villages de chefs sacrés appelés Tunkara

..... Limite du pays minyanka 
villages $^{\text {? }}$ et les nombreux interdits qui entravent l'existence de ces personnages font penser à l'institution du roi sacré.

On les appelle tangbula ou, dans la région de San, tunkara. Ce dernier terme évoque celui qui désignait le roi sacré du très ancien empire du Wagadou ou Ghana (3e-13e siècles) ${ }^{3}$.

A défaut d'avoir pu traduire ces deux titres, nous conviendrons d'appeler son titulaire "chef sacré". Il exerçait autrefois son pouvoir sur plusieurs villages et son comportement était censé affecter le cours du monde. Aujourd'hui, seul son village d'origine, où il a statut de chef de terre, lui reconnaît une autorité religieuse et la capacité d'influencer la fécondité.

Les Minyanka confèrent également les traits caractéristiques des chefs sacrés à des autels sacrificiels yapèrè placés sous le contrôle des chefs de culte (yapèrè folq). Confectionnés par les hommes pour maîtriser le monde, ces "objets-dieux" sont traités en souverains. Les Minyanka attribuent le titre des rois bambara masa aux puissances religieuses matérialisées par les yapèrè: nya, nankon, manyan, etc.

Les rites sacrificiels effectués sur ces autels sont l'occasion de rassemblements propices à l'évocation des problèmes politiques dont

\footnotetext{
2 Kadiala, Raména, Kéméni, Dougouolo, Bokoni, Yédala, Somaso, au nord de Mpessoba, dans l'arrondissement de Kimparana et dans les environs de San (Traoré, 1970). Ces villages sont contigus à ceux où siègent les rois sacrés maraka; voir carte.

${ }^{3}$ L'empereur du Ghana, le tunkara, était représenté au Mali par des chefs d'esclaves qui portaient également ce titre. Ces fonctionnaires avaient tendance à évincer les autorités locales qu'ils avaient pour mission de surveiller. Lors de la dislocation de l'Empire, la puissance de ces tunkara d'origine servile s'accrut. D'autre part, des princes tunkara refusant l'islam se réfugièrent vers le sud lors de la conquête des Almoravides à la fin du 1le siècle. Des clans sarakolé ou soninké originaires du Ghana émigrèrent dans toute la région soudanaise y diffusant leurs institutions et se fondant parfois aux Malinké. Lors de son voyage en Afrique occidentale au 19e siècle, E. Mage (1980: 58) rapporte qu'il rencontra près de Ségou un chef, très estimé chez les Soninké, portant le nom tunkara. Dans son étude sur les rois sacrés de la région de Ségou, J. Bazin (1988: 412 note 103) mentionne trois villages vers San, Daa, Ngwa et Sama, dont les chefs sont également appelés tunkara. Il note que l'ancêtre de cette dynastie Traoré aurait séjourné auprès d'un roi vers Sokoto ('Traoré, 1970: 34) dans l'ancien Etat de Méma, dont les souverains s'appelaient aussi tunkara. Or c'est effectivement dans cette région que des princes tunkara se sont réfugiés lors du démembrement de l'empire du Ghana.

4 Par cormodité pour le lecteur, les termes minyanka sont laissés au singulier. Le pluriel de yapèrè est yapèrlè.
} 
les plus délicats se règlent au nom des puissances religieuses. Les chefs de village ( $k u l u$ folg) gouvernent avec l'appui des sociétés initiatiques qui rassemblent les aînés des communautés lignagères ( gbuun folo), le chef de terre (ninge folq) ou le chef sacré ( $k u l u$ folo tangbula ou tunkara) ainsi que les chefs des sociétés initiatiques (yapèrè foll). Nous traduisons l'élément fọlo ou fo ( $\mathrm{pl}$. fè) par chef, mais ce mot met davantage l'accent sur le lien entre un individu et des personnes, des lieux ou des objets que sur la notion de direction ou de commandement (Colleyn-Jonckers, 1983).

La fonction de tous ces chefs est héréditaire, par ordre de primogéniture. Mais ils sont tous susceptibles d'être destitués s'ils ne font pas régner la prospérité et la paix autour d'eux. Ils doivent se montrer généreux et faire preuve d'un talent d'arbitre. Ils assurent le maintien de l'ordre, sans jamais monopoliser le pouvoir. lls exercent aussi des fonctions religieuses et se font respecter grâce à des connaissances occultes assimilables à de la sorcellerie (Colleyn, 1988: 85). Leur autorité ne repose pas sur la violence mais ils peuvent, le cas échéant, user de la force. L'autorité du chef sacré comme celle du manyan, l'un des plus puissants yapèrè, tend à dépasser le cadre du village alors que dans l'ensemble la société minyanka peut être qualifiée de villageoise (Capron, 1973: 65-66). Située dans une région périphérique des anciens royaumes de Ségou et de Sikasso, elle a été victime de ces Etats prédateurs mais n'a jamais été directement soumise à leur autorité.

Nous allons tenter de cerner le statut des chefs sacrés et des chefs de terre minyanka pour ensuite mettre en évidence la portée politique du culte du manyan et des sociétés initiatiques.

\section{Le chef sacré kulu folg tangbula ou tunkara}

Le chef tangbula ou tunkara, réputé descendant direct du premier occupant du village, doit être de père et de mère minyanka; il ne peut 
être d'origine peul ni descendant d'esclave, ni être né après le décès de son pères.

Le déroulement de son existence est censé affecter le cours du monde; dès lors tous ses actes sont soigneusement contrôlés et sa vie est réglée par des interdits et des devoirs. Il ne peut plus quitter le village; confiné dans sa case ou sous l'arbre à palabre, il n'est autorisé à sortir à la limite du quartier que la nuit pour satisfaire ses besoins. Personne ne peut s'adresser à lui publiquement; on ne peut lui parler que dans sa case, il doit couper les ponts avec ses parents et ses amis. Il ne peut plus entretenir de relations sexuelles avec une femme et doit s'abstenir de manger en public.

L'existence du chef sacré est tellement austère qu'un interrègne est prévu, dit-on, pour permettre à son successeur de prendre congé de la vie. Il est courant aujourd'hui que l'héritier refuse la charge en se convertissant à l'islam ou tout simplement en prenant la fuite.

Bien que la fonction soit héréditaire, le chef n'accepte pas facilement son rôle. Il est capturé par les membres d'une famille Keita d'origine mandé ${ }^{6}$. Dans le Mandé, les chefs soninké et malinké étaient des Keita. Seuls ceux-ci connaissent, aujourd'hui encore, les paroles de l'intronisation. Celle-ci se déroule en trois temps. La première partie appellée kulu folg tèngè (chef de village/asseoir) correspond à un simulacre de funérailles (Jonckers, 1975). Elle est suivie d'une réclusion de 42 jours pendant laquelle le chef, considéré comme mort, ne peut ni sortir de chez lui ni répondre à aucun appel venant du dehors.

\footnotetext{
$\therefore$ Les chefs sacrés tangbula de Somaso sont des Dao d'origine malinké et ils se rattacheraient à une des plus anciennes familles du Mandé, les Traoré.

" Le Mandé ou Manding qui englobe la Haute Vallée du Niger jusqu'en Guinée faisait partie de $l^{\prime}$ Empire du Ghana et fut le centre de l'Empire du Mali (13e-15e siècle) d'où émigrèrent les Malinké. Les familles Keita de Dougouolo et Somaso disent s'être installées dans ces villages au 11e siècle lors des mouvements de l'armée de Sunjata Keita dont ils faisaient partie. Cissoko (1966: 46) note que Sunjata était entouré de princes tunkara dans sa campagne contre la domination du roi Sosso qui s'était emparé du Ghana et des royaumes mandingues. Il signale (p.29) que Sunjata laissa son titre au roi du Ghana qui était respecté conme maître de la terre de son petit royaume musulman, scorie d'un glorieux passé.
} 
Enfin, lors du gnonton forglg (bonnet/sortir/dehors), le chef coiffé du bonnet (gnontonni), insigne du pouvoir royal, effectue une sortie publique. Les cérémonies commencent la nuit. Les chefs de quartier, aînés des familles fondatrices, emmènent le futur tangbula sur la place du village où il s'assied sur une natte face à l'est, appuyé contre une pierre ferrugineuse qui marque ce lieu sacré depuis la fondation de l'agglomération. Les musiciens récitent ses louanges, énumèrent sa généalogie et rappellent les migrations du Mandé.

Les anciens accompagnent ensuite le chef à la première cuisine des fondateurs du village, un des lieux consacrés aux ancêtres lignagers, pour la toilette mortuaire. Ils lui enfilent les vêtements blancs des morts et le ramènent sur la place publique pour la prise du bonnet. L'aîné des Keita pose le bonnet sur la tête du chef et proclame "Tout le village t'a choisi et te donne ce bonnet pour être son chef". Son épouse prend place à ses côtés, entièrement recouverte de couvertures blanches comme si elle portait le deuil de son mari. Désormais elle est effectivement considérée comme veuve et son mari renonce à tout rapport sexuel. Ce rite détache le chef sacralisé du monde des hommes. A l'aube, on le reconduit chez lui. Dès ce moment, il perd son nom et il ne peut plus y répondre sans risquer la mort. On doit l'appeler tangbula ou tunkara.

Les chants d'intronisation soulignent la pureté du chef, rappellent sa fonction de juge suprême et le caractère singulier d'un pouvoir qui s'appuie sur les puissances associées à la fondation du village.

"Que Dieu (Klè) t'aide

Que la vieille porte du village t'aide

Que le bois sacré du village t'aide

Que la jarre du village t'aide

Que le dasiri (animal protecteur) du village t'aide

Premier fils, chef de village

Que tu entendes cela

Ils t'ont fait tangbula

Tu es pur, pur 
Tu arrives à la fin de toutes choses

Dieu a fait ton nom semblable à lui

O notre premier fils de Dieu!

$\mathrm{Tu}$ es le bras en train de descendre sévèrement."

Ces paroles mettent en évidence la vieillesse et la proximité de la mort. En fait le tangbula est un ancêtre vivant. Il appartient au monde divin: il est le "premier fils de Dieu". La cérémonie de sortie de réclusion se déroule avec le plus de faste possible. Tous les villages reconnaissant l'autorité du tangbula contribuent au paiement de ses habits rituels et participent aux frais d'intronisation.

Le chef, toujours vêtu de blanc, sort de sa case, précédé d'un joueur de trompe et, suivi d'un vieillard portant une houe, il marche à reculons à l'instar des ancêtres qui se déplacent de cette façon dans le monde des morts. Ainsi comme chez les Rukuba du Nigéria (Muller, 1975 ), le roi commence son règne en assistant à sa propre mort symbolique afin de pouvoir régner le plus longtemps possible.

La suite du rituel d'intronisation se déroule à l'orée du village, au kan shoo tègè (le champ de la jarre du village) où un enclos de nattes, protégé par des hommes en armes, est dressé. Cet endroit est le lieu de fondation du village où une jarre témoigne des rapports harmonieux qui se sont établis entre les hommes et la terre. Le chef prend place dans cet abri aux côtés de son épouse, toujours drapée de blanc comme une veuve.

L'aîné des Keita aide le chef à endosser, par-dessus ses vêtements blancs, un grand boubou tissé, à petits carreaux noirs et blancs. Ensuite, par-dessus son bonnet blanc, il le coiffe d'un second couvre-chef de couleur rouge qui fait penser à celui que portait le roi sacré maraka (Bazin, 1988: 417). Pendant toute la journée, le chef reste assis dans l'enclos, sur la natte. A ses côtés sont déposés une canne en forme de serpent et un panier dans lequel les visiteurs déposent argent et cauris'. Tandis qu'ils présentent leurs salutations

\footnotetext{
7 Ces petits coquillages sont toujours utilisés comme monnaie lors des funérailles.
} 
au chef, les Keita se livrent à un vol rituel: ils dérobent tout ce qui est à portée de main dans le village.

Après cette cérémonie, le chef est autorisé à sortir de sa case mais il doit rester sur le territoire du village; il se déplace en compagnie de deux jeunes gens armés, chargés de défendre le bonnet, insigne du pouvoir. Autrefois, un village adverse pouvait tenter de s'en emparer et c'était le signal du déclenchement des hostilités ${ }^{a}$. Un village qui aurait perdu le bonnet et ne serait pas parvenu à le récupérer perdrait le droit de porter cet insigne et devrait se soumettre à son nouveau détenteur.

Le chef coiffé du bonnet et sacralisé perd sa liberté de mouvement; il devient "chose publique", véritablement assujetti à la société, dépouillé de sa personnalité propre. Comme le masa donta maraka de la région de Ségou (Bazin, 1988), comme le hogon dogon (Paulme, 1940; Griaule \& Dieterlen, 1954), le moger hadjeray (Vincent, 1975: 161) et le tyiri samo (Héritier, 1973), le tangbula est éloigné du commun des mortels.

Toujours présent au village, le tangbula doit être mis au courant de toutes les affaires. Il doit apaiser les conflits comme les rois maraka voisins mais à la différence de ces derniers, médiateurs impuissants qualifiés de "rois femmes" (Bazin, 1988: 381, 394, 395), les tangbula minyanka tranchent les litiges de façon irrévocable et celui qui s'oppose à ses décisions risque la mort.

Tandis que les modestes et pacifiques rois maraka étaient escortés par un vieillard ou un enfant, les tangbula minyanka étaient entourés de jeunes gens en armes. On ne leur décerne cependant pas le titre de roi (masa) que portaient leurs homologues maraka, les masa donta, mais celui de chef de village ( $k u l u$ folq), quand bien même leur pouvoir rayonnait sur plusieurs localités.

Le tangbula cesse de travailler; la communauté villageoise l'entretient; chacun doit lui donner une part de ce qu'il cultive: un panier d'environ vingt kilos par récolte et par famille. Personne ne peut le

\footnotetext{
A. Bazin, (1988:39), dans son étude sur les rois sacrés de la région de Ségou, signale que la possession du bonnet royal est un enjeu de guerre entre les descendants du très ancien royaume mandingue du Do (dissous vers le XVe siècle).
} 
saluer les mains vides car, dit-on, il est important de s'attacher sa bénédiction; "s'il vous l'accorde, Dieu vous l'accorde".

Dans les environs de San, le tunkara reçoit en outre les récoltes des champs cultivés par la population à son intention (Traoré, 1970: 40). Autrefois, les différents villages sur lesquels il exerçait son autorité devaient lui offrir chaque année cinq mille cauris, un mouton, le "prix de la boisson" et lui fournir des guerriers.

Le tunkara se distingue des autres chefs minyanka par l'ampleur des dons qu'il reçoit de ceux qui recherchent ses bénédictions et redoutent ses malédictions. C'est essentiellement le souci d'accroître la fertilité de la terre qui amène les Minyanka comme leurs voisins maraka (Bazin, 1988: 402) à faire de telles libéralités à leurs chefs sacrés.

\section{Le chef de terre ninge folg}

En dehors des régions septentrionales où domine la figure du tunkara, c'est le chef de terre, aîné du patrilignage des premiers occupants présumés du village, qui exerce la fonction rituelle. Il joue un rôle moins important que par le passé car bien souvent il n'exerce plus les fonctions de chef de village dévolues à un représentant du gouvernement malien. Mais il préside encore aux sacrifices au bois sacré (kashinge), à Dieu (Klè) et aux ancêtres (sefè). Ceux qui reconnaissent son autorité l'appellent toujours "chef de village".

$\mathrm{Au} 19 \mathrm{e}$ siècle, à l'époque où les guerres ravageaient le pays, il arrivait que des conquérants s'emparent d'un village et s'y installent. Le plus souvent, ils confiaient au chef d'une famille qui pactisait avec eux des fonctions d'intermédiaire. Fort de cette position, celui-ci disputait alors le pouvoir aux premiers occupants. Pour être reconnu, il devait néanmoins leur "acheter" la chefferie moyennant 100.000 cauris et des captifs (Collieaux, s.d.) ou deux filles et un cheval.

Dans certains villages, le dédoublement du pouvoir est dû aux colonisateurs français qui choisirent souvent dans la famille du chef de terre un homme qui leur était favorable. Lorsque le descendant 
des premiers occupants perd le contrôle de l'accès à la terre, les usurpateurs continuent néanmoins à faire appel à lui pour les sacrifices au bois sacré qui appellent la pluie. L'un des titres du chef de terre est d'ailleurs kashikan folo, "chef du sacrifice au bois sacré" ou wèrè folo, "chef des plantes", en sa qualité de connaisseur des plantes (wèrè) qui entrent dans la composition des remèdes, des poisons et des autels yapèrè.

Un cultivateur non musulman qui veut défricher un champ s'adresse au chef de terre pour se concilier les forces chthoniennes. Il attend de lui le signal qui lui permettra d'effectuer les travaux agricoles sous des auspices favorables. Chaque année les chefs de famille respectueux des traditions offrent au chef de terre quelques épis de mil et de maïs. Ces dons permettent à ce dernier de faire face aux sacrifices collectifs.

Dans les villages à majorité musulmane, où le chef administratif est lui-même islamisé, l'autorité du chef de terre s'affaiblit car son rôle spirituel est amoindri. Mais le plus souvent, tous deux sont, à leur manière, garants de l'ordre social et arbitres en cas de litige. Leur autorité ne s'exerce pas directement sur chaque individu mais sur les chefs des communautés familiales qui sont responsables de leurs dépendants.

Le chef de terre est le serviteur rituel du village; il ne peut s'absenter car les sacrifices qui déterminent le bien-être collectif, c'est-à-dire la paix, l'entente, la fécondité des femmes, la venue de la pluie et la fertilité des sols lui incombent. J.F. Vincent (1975: 173) dit très justement du chef de terre hadjeray, dont le statut et les fonctions sont semblables à ceux de son homologue minyanka, qu'il est un "catalyseur de fertilité" dont la personne physique, voire l'humeur, sont liées à la prospérité des champs.

Les Minyanka se préoccupent de la santé du chef de terre car elle reflète le bien-être de la communauté. Si ce chef est cloué au lit par la maladie, il doit pouvoir se lever pour procéder aux sacrifices au moment opportun. S'il n'y parvient pas, il en meurt.

Nous avons assisté à un sacrifice septennal de purification du village. Le chef de terre malade n'a pu se lever. Son successeur dirigea le rituel. Le même jour, le chef qui avait failli à sa tâche 
mourut. Il ne fait de doute pour personne que, dans de telles circonstances, la mort est inévitable.

Jadis, lorsque la guerre était courante, il revenait au chef de terre d'exécuter les sacrifices au bois sacré lors du départ et du retour des expéditions. Les Minyanka n'évoquent pas volontiers ces pratiques car il s'agissait de sacrifices humains. Ces rites conféraient au chef de terre un caractère hors du commun. Aujourd'hui, il vit très humblement comme un saint homme mais on lui reconnaît toujours une personnalité particulièrement forte.

Normalement les chefs de village et les chefs de terre sont essentiellement des pacificateurs (susuro) et ils mettent tout en oeuvre pour éviter les affrontements, réparer les dommages et, le cas échéant, exiger des sacrifices expiatoires. Toutefois, ils avaient jadis le pouvoir d'appliquer des sanctions; celles-ci allaient de l'auto-critique publique (pour vol de mil ou de bois) à la peine de mort (pour un crime) ou au bannissement (pour un inceste) en passant par les châtiments corporels (à coups de corde) et la mise aux fers (pour coups et blessures). Mais c'est la crainte de la réprobation qui était (et demeure) le principal régulateur de l'ordre social. Néanmoins les Minyanka ont tendance à admirer ceux qui réussissent à imposer une certaine violence et qu'ils appellent les hommes forts, fah folg.

\section{Les hommes forts fah folg}

Des hommes entreprenants ambitionnaient parfois d'acquérir le titre de fah folg en tuant un étranger après avoir sacrifié un poulet sur les autels de la guerre, notamment sur le manyan.

Il n'existait pas de plus haute source de renom que de risquer un coup de main contre un village voisin. Les hommes aimaient se battre et montrer qu'ils avaient le "coeur dur" ( $n$ 'so wah). Jusqu'au 19e siècle, les agressions contre les voisins étaient considérées comme des actes de bravoure et témoignaient du pouvoir magico-religieux des puissances invoquées. Les escarmouches, les conflits de prestige et autres volées de flèches ne répondaient à aucune tentative d'expansion 
territoriale ni de centralisation étatique. Le chef de terre-chef de village ou les chefs de culte arbitraient les conflits et procédaient aux sacrifices nécessaires.

Les menaces d'agression rendaient chaque déplacement fort dangereux. Avant de partir en voyage, l'on offrait des sacrifices aux puissances religieuses. Celles-ci étaient aussi bien invoquées pour se protéger que pour attaquer. Le manyan était, à cet égard, fort réputé et on sacrifiait sur ses autels au départ comme au retour de la guerre. Il jouait cependant un rôle pacificateur dans la mesure où les villages pratiquant ce culte ne pouvaient se combattre. Lorsqu'un village était attaqué, il pouvait arriver que le fah folg se mue en chef de guerre (kashi folg) en rassemblant des guerriers au sein d'une association volontaire (ton). Les associés (ton den) se soumettaient à l'intelligence, à la chance de leur leader, et à son habileté à constituer des coalitions. Ils admiraient aussi sa générosité. Ces qualités purement personnelles ne sont pas héréditaires. En principe i'autorité d'un "homme fort" n'était acceptée que de manière ponctuelle. Jamais il ne devenait chef de village.

Les Minyanka partagent ici la conception des Bambara (Bamana) ${ }^{9}$ qui opposent le pouvoir héréditaire sacré au pouvoir militaire contingent fondé sur le principe de l'association (Bazin, 1982: 7). Ils donnent à ces hommes forts le titre bambara faama (de fanga ma, dépositaire de la force) qu'on octroie aux rois guerriers et par extension aux personnes riches ou puissantes.

Si ce type de pouvoir est conjoncturel, il arrivait cependant qu'un chef de ton se maintienne en place par la force et transforme ses associés en ton jon, "esclaves du ton". Ces prises de pouvoir étaient chose courante dans l'ambiance des Etats guerriers du 19e siècle et elles ne sont pas étrangères à la vie politique actuelle (Jonckers, 1987). C'est ainsi que certains chefs de village n'hésitent pas à monopoliser l'association des cultivateurs (ton), voire à utiliser les fonds de l'association villageoise (AVE) à leur profit personnel.

\footnotetext{
"Rappelons que les Minyanka de même que leurs voisins dyonka et senufo revendiquent le nom de Bamana en dépit de l'usage qui réserve ce terme aux Bambara (Bazin, 1985).
} 
D'aucuns ont même livré à la gendarmerie des chefs de famille incapables de payer l'impôt alors qu'il est traditionnellement de leur devoir d'aider les villageois en difficulté. Ces chefs ne parviennent à imposer leur pouvoir que par la violence en s'appuyant sur la menace d'une intervention de l'Etat central.

Les Minyanka confèrent aussi un pouvoir de coercition indirect aux détenteurs d'autels sacrificiels yapèrè et aux puissances religieuses. Celles-ci portent d'ailleurs à la fois le titre bambara de faama qui révèle leur caractère redoutable et celui de masa qui les assimile à des rois sacrés pacifiques. Dans la région de Ségou, masa, sans autre précision, fait généralement penser à la souveraineté sacrée dépourvue d'appareil de contrainte (Bazin, 1988: 378-79). Il est remarquable que les yapèrè mịnyanka cumulent les caractères du faama et du masa. Il en est ainsi du manyan.

\section{Le culte du manyan et le rôle politique des sociétés initiatiques}

L. de Heusch (1986: 271) a suggéré que la puissance surnaturelle nya représentée par un objet-fétiche du même nom et qui se trouve au centre d'un important culte de possession présente certaines caractéristiques du roi sacré. Nous allons montrer qu'il en est de même d'une autre puissance, le manyan, représenté par d'importants autels.

Aujourd'hui, le manyan n'est plus guère invoqué pour les questions guerrières; il est surtout sollicité pour favoriser la fécondité des femmes et la fertilité du sol. On attend de lui qu'il accorde des pluies régulières et qu'il prévienne les catastrophes naturelles. Á cette fin on lui sacrifie de nombreux animaux. Selon le mythe, c'est un chasseur qui serait à l'origine du culte, alors qu'un guerrier est le plus souvent le héros de l'histoire humaine. Ceci dit, chasseur et guerrier, êtres extérieurs à la communauté villageoise, détiennent une puissance particulière leur permettant de se risquer à détruire la vie (celle des animaux ou des hommes). Tous deux connotent dans une certaine mesure une rupture avec l'ordre lignager. C'est au chasseur que revient 
le soin de rechercher de nouveaux territoires au moment où la segmentation s'impose, et la vocation de guerrier tente ceux qui se sentent à l'étroit dans les structures lignagères.

Les premiers chefs de culte dont les Minyanka ont conservé la mémoire sont en général des hommes puissants, dotés d'une force guerrière peu commune, réputés pour leur hyperpolygamie (plus de deux cents femmes) ou connus comme familiers des rois de Ségou et de Sikasso. On les qualifie de fah fo ou de faama.

Les autels du manyan dégagent eux-mêmes une impression de force. Ils ont la forme de cônes et de sphères, recouverts d'une épaisse croûte de sang coagulé et ils sont parfois tellement volumineux qu'on ne peut les déplacer. Ils sont entourés d'objets symbolisant la puissance: un cor de guerre (originaire de Ségou où il était utilisé pour rassembler les guerriers royaux) et des armes (la hache, l'arc et les flèches, le fusil et surtout la lance, marque de distinction des rois de Ségou) (Monteil, 1924). Trois autres composantes du manyan sont également des signes des rois de Segou (masa): le sceptre bèri, la queue de vache blanche, le chasse-mouche, et la tenue rouge vif ${ }^{\circ}$. On recouvre en effet le manyan d'étoffes rouges lors des cérémonies. Car c'est bien en roi sacré que l'on traite le manyan lors des rites sacrificiels, les dignitaires du culte ôtent leurs chaussures et leur chapeau en signe de respect avant de s'agenouiller tête baissée devant le sanctuaire pour accueillir le manyan.

Les autels, acclamés par la foule, sont sortis à reculons du sanctuaire à l'instar des ancêtres dans l'univers des morts. On les attache ensuite sur un brancard mortuaire et on les enveloppe d'un suaire: couverture blanche des défunts ou plus souvent drap rouge des masa. Ces rites ressemblent à ceux de l'intronisation du roi sacré bambara et du chef sacré minyanka qui assistent au simulacre de leurs funérailles. Tandis que les grands autels côniques posés sur des brancards funéraires sont portés sur la tête, les dignitaires arborent

\footnotetext{
${ }^{10}$ Selon Monteil (1929: 132, note 1), la couleur rouge était la couleur préférée des chefs du Mali. Déjà du temps du lointain Gnana on importait d'Espagne des blouses en étoffe rouge. Les cortèges royaux comportaient des groupes d'esclaves vêtus de rouge et des drapeaux rouges étaient brandis. Cet usage persista chez les chefs bambara.
} 
les armes du manyan. Comme les rois de Ségou, les autels du manyan se déplacent toujours en compagnie d'un forgeron.

Les initiés appartenant à des villages différents participent aux cérémonies de plusieurs localités et entretiennent des relations suivies. Il se crée ainsi des sortes de "fédérations villageoises" où se nouent des alliances guerrières et des accords de chasse comme on l'observe également chez les Samo autour des maîtres de la pluie ( Fr. Héritier, 1973). Il y a des interférences entre ces différentes alliances créatrices de zones de paix et le tissage des liens matrimoniaux (Jonckers, 1990). On a peut-être trop insisté sur le caractère autonome des villages. Certes ceux-ci revendiquentfarouchement leurindépendancepolitique, mais cela ne signifie pas qu'ils vivent totalement repliés sur eux-mêmes .

Alors que le manyan est honoré comme un puissant roi sacré, on en parle comme s'il s'agissait d'une femme. Le chef de culte considère le manyan comme son épouse et l'acquisition des autels est assimilée à un mariage (Jonckers, $1986 \mathrm{a}$ ).

Marc Augé (1988: 55) insiste sur le fait que le "dieu-objet" se présente comme un corps même lorsqu'il ne peut être vraiment dit anthropomorphe. Le "dieu-objet" est sexué, il se reproduit et doit être nourri. Ainsi l'autel principal du manyan s'appelle "vieille mère" (ba koro) et est indispensable à la reproduction des autels qui se réalise selon une filiation utérine (Jonckers, 1986 b). Chaque "autel fille" comprend une parcelle de cet "autel mère". Néanmoins figure à ses côtés un autre autel qualifié de "premier fils", zye, qui représente l'autorité masculine. On y trouve aussi les armes de chasse et de guerre relevant exclusivement de l'univers masculin.

Si on s'adresse le plus souvent au manyan comme à une femme, on le loue cependant comme s'il était un homme, puisqu'on le qualifie de "vieil homme puissant". Nous avons donc affaire à vrai dire à une puissance bisexuelle.

Ce caractère androgyne est l'apanage des dieux dans l'aire mandé mais il peut aussi être interprété comme le reflet des deux facettes du pouvoir royal du manyan: l'une masculine usant de la force sur 
le modèle du faama, et l'autre féminine et magique comme celle du masa (Bazin, 1988: 379).

Mais tout dieu ou roi sacré qu'il soit, le manyan doit être nourri sous peine de mourir. On enduit soigneusement les autels du sang des animaux sacrifiés et on y écrase le foie et le coeur des victimes. Les initiés partagent ce repas en consommant la chair des animaux sacrifiés.

Le plus souvent on évoque le manyan pour lui demander des enfants. Celui qui acquiert ces autels assure la fécondité de son lignage et celle des membres de la confrérie qu'il fonde. La plupart des naissances dans un quartier où est implanté le culte lui sont attribuées et les enfants natifs du lieu, appelés "enfants du quartier" (kapia), doivent participer aux sacrifices annuels au manyan.

Le pouvoir du manyan sur la nature (l'un des traits essentiels de la royauté sacrée) porte non seulement sur la fécondité mais aussi sur le contrôle de la pluie ou du soleil, comme l'évoque ce chant:

"Tu peux être soleil et sécher les choses

Tu peux être pluie et faire germer les semences"

Il y a bien des points communs entre le statut du manyan et celui des chefs sacrés tangbula (ou tunkara) ou du chef de terre. Ces personnages sont censés détenir eux aussi des pouvoirs sur la nature, et leur vie est entourée d'interdits à tel point qu'on peut les considérer comme des objets-fétiches.

Le destin du chef de culte est étroitement lié à celui du manyan et il ne peut renoncer à ses fonctions sous peine de mort. Comme le chef sacré, ce chef est soumis à des interdits: il ne peut entretenir de rapports sexuels avec ses épouses les jours où il se consacre au manyan ni s'absenter plus de deux jours consécutifs du village.

Les porteurs du manyan, désignés par un sacrifice divinatoire, ne peuvent non plus renoncer à leur charge. Lorsqu'ils sont trop âgés pour s'en acquitter, ils deviennent sacrificateurs. A l'époque où le transport des autels était effectué par des esclaves, ceux-ci étaient mis à mort en vieillissant ou à la moindre défaillance et leur coeur, comme celui des victimes sacrificielles, était écrasé sur les autels. 
La notoriété du chef de culte peut dépasser le cadre villageois, mais il n'exerce pas pour autant un pouvoir personnel. Les différentes fonctions rituelles (musicien, sacrificateur, bouffon) sont toujours réparties entre différents lignages.

D'une manière générale, le pouvoir magique des chefs de culte les contraint à ne pas s'éloigner du village et à se soumettre à certains interdits, notamment à l'abstinence sexuelle avant un sacrifice. Néanmoins ces chefs ne possèdent pas dans leur être le pouvoir sur la nature qu'on attribue au chef sacré, dont la liberté de mouvement est totalement entravée, et au chef de terre dont la santé est l'objet d'une attention constante. Leur autorité reflète la puissance de l'entité religieuse qu'ils servent et repose sur le fait qu'ils sont les intercesseurs obligés des hommes auprès de celles-ci. En fait ces chefs de culte sont perçus comme les représentants d'une puissance redoutable et à ce titre ils participent au maintien de l'ordre lignager en se fondant sur une force contraignante immatérielle.

Ils ne sont respectés que dans la mesure où la paix ainsi que la prospérité économique et sociale règnent dans la communauté villageoise. Si le village périclite, si la sécheresse sévit, si les récoltes sont mauvaises, si de nombreuses disputes éclatent, la faute leur en incombe. C'est la preuve que les sacrifices qu'ils offrent aux divinités et aux ancêtres ne sont pas acceptés. Après quelques années de malchance, un chef de culte renoncera à ses fonctions et abandonnera ses autels, d'ailleurs désertés par les initiés au profit d'autres plus efficients .

Les chefs de famille, porte-parole de leurs dépendants auprès des puissances religieuses et des ancêtres, peuvent également être destitués si leur famille connaît des difficultés pendant plusieurs années consécutives. L'aîné tenu pour responsable de la situation est alors écarté au profit d'un cadet. C'est le possédé qui règle de tels problèmes lors des rites sacrificiels. 


\section{Conclusions}

Dans cette société où les fonctions politiques se répartissent entre plusieurs acteurs, la symbolique du pouvoir sacré est en oeuvre à divers niveaux. Les chefs de terre, les chefs de culte et les chefs sacrés proprement dits ainsi que les "objets-fétiches" dont le manyan sont censés détenir un pouvoir sur la nature grâce à une puissance magique. On leur reconnaît la capacité de lutter contre les sorciers et de contrôler les facteurs naturels comme la pluje et la fécondité dont dépend l'existence de la communauté.

La puissance magique et l'emprise sur la nature fondent la sacralité de leur pouvoir. Celle-ci est plus ou moins marquée selon qu'ils sont eux-mêmes dépositaires de ce pouvoir magique comme l'objet-fétiche manyan, le chef sacré et le chef de terre ou qu'ils ne sont que des intermédiaires auprès de puissances mythiques comme les chefs de culte.

Un réseau d'interdits frappe ces "rois sacrés en puissance": leur vie sexuelle est marquée par des périodes d'abstinence, leur liberté de mouvement est limitée et ils sont plus ou moins assignés à résidence car leur comportement est censé affecter le cours du monde. Leur statut rappelle celui du roi divin défini par Frazer (1920), à la différence près que ces autorités ne sont pas mises à mort rituellement. Néanmoins elles subissent une mort symbolique: le chef sacré assiste à un simulacre de ses funérailles lors de son intronisation, le chef de terre meurt si la maladie l'empêche d'exercer ses fonctions sacrificielles, et les chefs de culte sont abandonnés par leurs fidèles si la prospérité et la fécondité ne règnent pas dans la communauté. Quant au manyan on peut considérer qu'il est un chef sacré fétichisé dont on rejoue l'intronisation, semblable elle aussi à des funérailles, lors des rites sacrificiels .

La diversité des modèles politiques minyanka est liée aux brassages de populations qu'a connus cette région située à l'orée des anciens empires de l'Afrique de l'Ouest, le Ghana ou le Mali et, plus récemment, les Etats de Ségou et de Sikasso.

Les Minyanka relèvent culturellement de l'aire mandé et, bien qu'ils parlent une langue senufo, ils utilisent de nombreux termes 
bambara, notamment dans le domaine politique. Certains rites comme l'intronisation du chef sacré et le culte du manyan évoquent les traditions bambara et maraka. Il est d'ailleurs probable, ainsi que le suggèrent J.L. Amselle (1990: 194) et J. Bazin (1988), que ces institutions assuraient l'intégration des sociétés périphériques (les Minyanka en l'occurrence) aux grands ensembles politiques.

On remarquera aussi dans d'autres sociétés, où l'au torité concerne essentiellement le groupe local, certains traits des royautés sacrées voisines. C'est le cas des populations gurunsi (Capron, 1973: 5457; Dittmer, 1961; Duperray, 1984) voisines du royaume mossi du Burkina Faso, des Hadjeray (Vincent, 1975: 161) du Tchad, ou des Rukuba (Muller, 1980: 160-161) proches du royaume jukun (Meek, 1931) du Nigéria. Les petites chefferies pende et lele (de Heusch, 1987a: 289) s'inscrivent également dans un continuum structural où figurent les royaumes lunda et kuba du Zaïre.

Les formes de pouvoirs sacrés ont vraisemblablement été diffusées par des fonctionnaires locaux qui se sont affranchis de la tutelle du pouvoir central, ou par des princes en rupture de ban. Les modestes rois faiseurs de paix maraka seraient les descendants des roitelets vassaux de l'Empire du Mali et les tangbula minyanka se seraient établis lors des campagnes militaires de Sunjata Keita. Mais, comme le remarque Bazin (1988: 413, n. 108), il n'est pas impossible que des chefs locaux se soient "mandinguisés" au contact des Keita du Mandé avec lesquels ils guerroyaient.

Les chefs minyanka et le manyan, dotés de pouvoirs magiques relativement semblables, offrent deux images différentes du pouvoir sacré. Les chefs sont sacralisés indépendamment de l'étendue de leur pouvoir politique. Celui-ci peut dépasser le cadre villageois mais cette extension ne prélude pas à la formation d'un Etat car ces chefs contribuent essentiellement au maintien de l'ordre lignager et à la gestion communautaire du village. Nous rejoignons ici les conclusions d'Alfred Adler (1977: 56-57) à propos du lamutyiri samo.

Ces chefs n'échappent pas au modèle lignager selon lequel seuls les hommes âgés exercent l'autorité. Ils ne disposent pas de la force 
coercitive; c'est la menace d'une punition surnaturelle et la désapprobation publique qui suffisent à maintenir l'ordre. Les chefs de culte, assistés dans certains cas des possédés, jouissent d'un pouvoir politique non négligeable car ils résolvent les problèmes et exécutent les sentences éventuelles en faisant appel à l'opinion de la population, mais leurs injonctions ne sont suivies que si elles font l'objet d'un consensus. Ils ne peuvent orienter la vie politique du groupe qu'avec l'assentiment de celui-ci. En revanche les puissances invisibles (telles le manyan) sont susceptibles de tuer.

Les chefs se doivent d'être généreux et ne peuvent rien exiger de ceux qui reconnaissent leur autorité. C'est en vertu de leurs fonctions rituelles qu'ils reçoivent des dons plus ou moins importants. Ainsi le chef sacré tangbula est totalement pris en charge par ses sujets; le chef de terre reçoit un hommage sous forme de céréales tandis que le manyan est gratifié de nombreux sacrifices (le même terme désigne en minyanka le sacrifice et le don).

L'autorité de la plupart des chefs ne repose pas sur la coercition. Cependant le chef sacré tangbula comme le chef de terre pouvaient appliquer diverses sanctions et même la peine de mort. L'exercice d'une autorité sans partage est une idée étrangère aux Minyanka. Lorsqu'ils l'évoquent, c'est à propos du royaume de Segou. On remarquera aussi que ce n'est pas la force physique qui ouvrait la voie au pouvoir puisque les "hommes forts" ( $f a h$ folg) se voyaient interdire la chefferie villageoise. Aujourd'hui les pouvoirs du chef de terre, comme ceux du chef sacré tangbula se sont effacés. Le manyan lui-même a généralement disparu, mais là où il se maintient, son rayonnement est considérable et l'on voit même des hommes politiques et des commerçants puissants (assimilés à des faama) solliciter son appui.

Danielle Jonckers URA 221 - (EPHE-CNRS) 


\section{Références bibliographiques}

Adler A.

1977 "Faiseurs de pluie, faiseurs d'ordre", Libre, 2, Payot, 4568.

Amselle J.J.

1990 Logiques métisses. Anthropologie de l'identité en Afrique et ailleurs, Paris, Payot, (Bibliothèque scientifique), 257p.

Augé M.

198 Le dieu-objet, Paris, Flammarion, $148 \mathrm{p}$.

Bazin J.

1975 "Guerre et servitude à Ségou", in C. Meillassoux éd., L'Esclavage en Afrique précoloniale, Paris, Maspero, 135182.

1982 "Etat guerrier et guerres d'Etat", in Guerres de lignages et guerres d'Etat en Afrique, J. Bazin et E. Terray, Paris, Archives Contemporaines, 321-372.

1985 "A chacun son Bambara" in Au coeur de l'ethnie, Amselle J.L. et M'Bokolo, La Découverte ('Textes à l'appui), Paris, 87-126.

1988 "Les 'rois-femmes' de la région de Segu", Cahiers d'Etudes Africaines. Manding, 111-112, XXVIII, 3-4, 375-442.

Capron J.

1973 Cornmunautés villageoises bwa. Mali, Haute-Volta, Paris, Institut d'Ethnologie (Musée de l'Homme), $379 \mathrm{p}$.

Cissoko S.M.

1966 Histoire de l'Afrique occidentale. Moyen-âge et temps modernes, VIIe siècle-1850, Paris, Présence Africaine, 333p.

Colleyn J.-P.

1988 Les chemins du Nya. Culte de possession au Mali, Anthropologie visuelle, Paris, EHESS, $221 \mathrm{p}$.

Colleyn J.P. et Jonckers D.

1983 "Les Minyanka 'ceux qui refusent le maître"", Africa, LIII, $4,43-58$.

Collieaux

s.d. Contribution à l'étude du Kénédougou, Archives Nationales de Bamako, Mali. 
Dieterlen G.

1973 "L'image du corps et les composantes de la personne chez les Dogon", in La notion de personne en Afrique noire, Paris, Acte du colloque, CNRS, 205-229.

Dittmer K .

1961 Die Sakralen Haüptlinge der Gurunsi in Ober-Volta Gebiet, Halburg, Kommissions Verlag Cram, de Gruyter and Co.

Duperray A.M.

1984 Les Gourounsi de Haute-Volta, Stuttgart, Franz Steiner Verlag Wiesbaden, Studien zur Kulturkunde, $281 \mathrm{p}$.

Evans-Pritchard E.E.

1968 Les Nuer. Description des modes de vie et des institutions d'un peuple nilotique, (trad. fr. L. Evrard), Paris, Gallimard.

Frazer J.G.

1920 Les origines magiques de la royauté, Paris, Geuthner.

Griaule M. et Dieterlen G.

1954 "The Dogon", in African Worlds, Studies in the Cosmological Ideas and Social Values of African peoples, International Arican Institute, Oxford University Press, 83-110.

Héritier F .

1973 "La paix et la pluie. Rapports d'autorité et rapport au sacré chez les Samo", L'Homme, 3, 121-138.

Heusch L. de

1962 "Pour une dialectique de la sacralité du pouvoir" et "Aspects de la sacralité du pouvoir en Afrique", in Le pouvoir et le sacré, Institut de Sociologie, Université Libre de Bruxelles, 15-48 et 139-158.

1986 Le sacrifice dans les religions africaines, Paris, Gallimard, $354 \mathrm{p}$.

1987a Ecrits surlaroyauté sacrée, Institut de Sociologie, Bruxelles, Université Libre de Bruxelles, $314 \mathrm{p}$.

$1987 \mathrm{~b}$ "L'inversion de la dette (propos sur les royautés sacrées africaines)", in M. Abensour, L'esprit des lois sauvages, Paris, Seuil, 41-57.

Jespers $\mathrm{Ph}$.

1976 "Contribution à l'étude des autels sacrificiels du Nya chez les Minyanka du Mali", Systèmes de pensée en Afrique noire, II, 111-139. 
Jonckers D.

1975 "Eléments ethnographiques: les rites funéraires chez les Minyanka du Mali", Systèmes de pensée en Afrique noire, I, 35-52.

1986a "Les faiseurs d'enfants. Réflexions sur le statut des femmes dans le système religieux minyanka", Journal de la Société des Africanistes, LVI, 1, 51-66.

1987 La société minyanka du Mali, Paris, L'Harmattan, (Connaissances des hommes), $234 \mathrm{p}$.

1990 "Hiérogamie et stratégies matrimoniales" in Les complexités de l'alliance, F. Héritier éd., vol.1, Paris, Archives contemporaines (Ordres sociaux).

Mage E.

1980 Voyage au Soudan occidental (1863-1866), Paris, Karthala (1ère éd. 1872), $308 \mathrm{p}$.

Meek C.K.

1931 Tribal Studies in Northern Nigeria, Londres, Kegan Paul, Trench, Trubner et Co Ltd, $633 \mathrm{p}$.

Monteil Ch.

1924 Les Bambara de Ségou et de Kaarta, Paris, Larose.

1929 Les Empires du Mali, Paris, Maisonneuve et Larose, 157 p.

Muller J.-C.

1975 "La royauté divine chez les Rukuba" (Benue Plateau State, Nigeria), L'Homme, XV, 1, 5-27.

1980 Le roi bouc émissaire. Pouvoir et rituel chez les Rukuba du Nigeria, Québec, Serge Fleury/Paris, L'Harmattan.

Paulme D.

1940 Organisation sociale des Dogon, Paris, Domat-Montchrestien, $603 \mathrm{p}$.

Tarikh-el-Fettach

1981 Tarikh-el-Fettach ou chronique du chercheur pour servir l'histoire des villes, des armées et des principaux personnages du Tékrour, trad. par $\mathrm{O}$. Houdas et M. Delafosse, Paris, Maisonneuve et Larose (1ère éd. 1913-14).

Terray E.

1989 "Une nouvelle anthropologie politique", L'Homme, CX, XXIX, 2, 5-29.

Traore El Hadj S.

1970 Notes sur le Nadougou, Bulletin de l'IFAN, 126, 33-41.

Vincent, J.F.

1975 Le pouvoir et le sacré chez les Hadjeray du Tchad, Paris, Anthropos, $226 \mathrm{p}$. 